\title{
The results of the vine varieties reconversion measures in the North-Eastern Romania / Résultats de la mesure de reconversion des cépages dans le Nord-Est de la Roumanie
}

\author{
Florin-Alexandru Enia ${ }^{1,2}$ \\ ${ }^{1}$ Laboratory UMR ARTeHIS, University of Burgundy, 21000 Dijon, France \\ ${ }^{2}$ Laboratory Geomatics, University A. I. Cuza, 700506 Iaşi, Romania
}

\begin{abstract}
The vine varieties reconversion measure is one of the many benefits that the Romania's integration to the European Union brings to the wine sector. This study aims to analyze the results of this measure during 2007-2015 in the Romania's North-East Region. From the Vineyard Plantation Register, the plots noble vine parcels of each department were divided into two groups: those who existed in the field in before and those converted during the period. The surface of each group was classified first, based on the color of the grapes and secondarily, based on the pair international varieties - non international varieties. The conversion affected 3 of 6 departments. In Iaşi, where the proportion of the surface of white grapes approach to $90 \%$ in 2006, the conversion was made whit white grapes ( $81.3 \%$ of the surface), and particularly whit 4 local varieties corresponding to the traditional assortment of the Cotnari vineyard. In Vaslui, the conversion was made particularly with white grape varieties, but below the proportion held in 2006, because the conversion was made whit a large area of rosé varieties (19.2\% of the surface) following the consumer demand. With the exception of Bacău, in the other two departments, the non-international and local varieties were used for the conversion.
\end{abstract}

\section{Introduction}

La Région de développement de Nord-Est est la plus entendue région de Roumanie, occupant une surface de $36850 \mathrm{~km} 2$, soit $15,46 \%$ de la surface du pays. De point de vue administratif et territorial, la région est divisée en 6 départements : Bacău, Botoşani, Neamţ, Iaşi, Suceava et Vaslui (Fig. 1). La population d'à-peu-près 3,7 millions d'habitants $(17,25 \%$ de la population du pays) vive principalement dans le milieu rural $(56,6 \%)$. La population occupée travaille plutôt dans le secteur agricole $(42,7 \%)$, suivi par le secteur des services $(37,9 \%)$ et par l'industrie $(19,4 \%)$ [1]. Le relief de la région comprend des montagnes (30\% de la surface totale), des sousmontagnes (30\% de la surface totale) et le plateau (40\% de la surface totale). A l'ouest il y a des montagnes ayant des altitudes de plus de 2000 mètres. Au nord-est il y a la Plaine Collinaire de la Moldavie, tandis qu'au sud-est de la région il y a le Plateau de Bârlad [2].

$\mathrm{Au}$ développement de la pratique viticole dans la région ont contribué les conditions favorables de l'environnement, mais également la tradition de la population de valoriser les versants impropres aux autres cultures [3]. La pratique de la viticulture date d'avant de l'époque des Daces. La première attestation documentaire des vignes sur ce territoire date de 1384. Le document appartenant au voïévode Petru Muşat fait référence aux vignes près de la localité de Hârlău du vignoble de Cotnari [4].

En ce qui concerne les conditions physicogéographique, au nord et au centre il y a des marnes, des grès calcaires et des calcaires qui construisent des plateaux de 400-500 mètres. Au sud il y a du sable et du lœss disposés dans des collines de 200-450 mètres et des plaines tabulaires de 50-200 mètres. Le climat tempéréecontinental peut donner approximativement 120 jours avec des températures moyens au-dessous de $0^{\circ} \mathrm{C}$ et même des températures minimales au-dessous de $-18^{\circ} \mathrm{C}$ pouvant poser des problèmes à la vigne. Dans la partie nord-ouest de la région, la température moyenne annuelle est de $7,5^{\circ} \mathrm{C}$ et les précipitations arrivent à $600 \mathrm{~mm}$ par an. Dans la partie sud-est, la température moyenne annuelle est de $10^{\circ} \mathrm{C}$ et les précipitations arrivent à $400 \mathrm{~mm}$ par an. Les sols font partie de la classe des luvisols et des tchernozioms [3].

Les plus importantes vignobles de la Région de NordEst sont situés dans la moitié est. Dans le département de Iaşi il y a les vignobles de Cotnari et de Iaşi. Dans le département de Vaslui, il y a le vignoble de Huşi. A l'exception des vignobles, il y a aussi des centres viticoles indépendants comme Hlipiceni, Plugari, Probota et Vaslui [5] (Fig. 2).

L'article présent propose une analyse sur les résultats de la mesure de reconversion des cépages dans la Région de Nord-Est, pendant la période 2007-2015.

La structure de cet article comprend 4 parties : introduction, données et méthodologie, résultats de l'analyse, conclusion.

\section{Données et méthodologie}

Il a été utilisé les données sur les parcelles en vignes nobles, dans les départements de la région. Ces données ont été extraites et fournies du Registre des Plantations Viticoles (R.P.V.) qui est sous la gestion de l'Office 


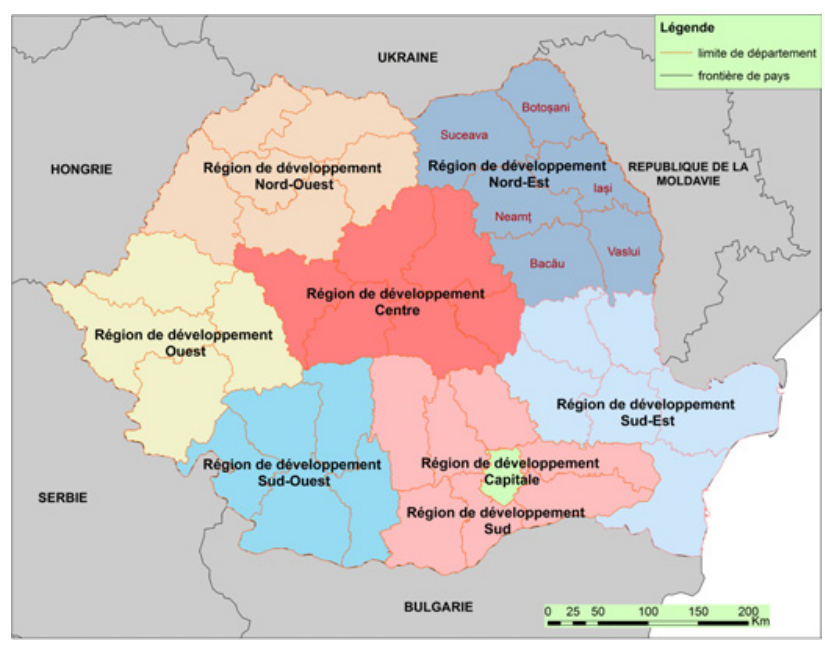

Figure 1. L'encadrement géographique de la Région de Nord-Est (carte d'auteur).

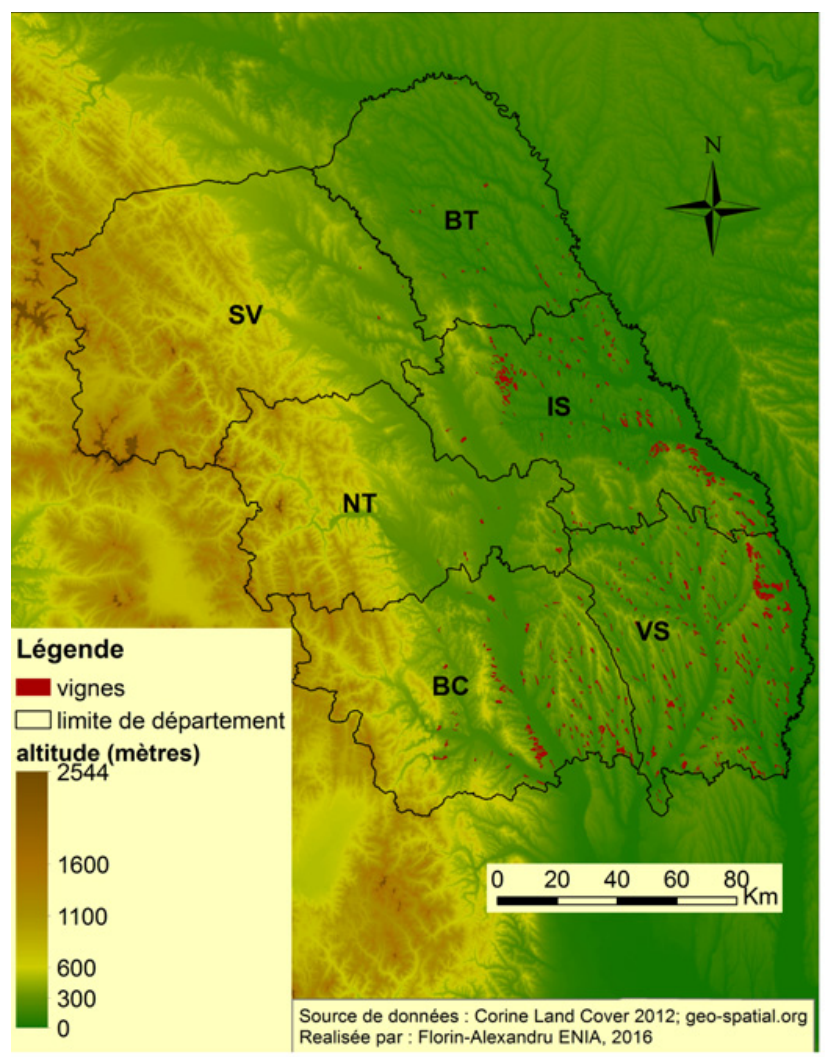

Figure 2. Dispersion des vignes dans les départements de la Région de Nord-Est en 2012.

National de la Vigne et des Produits Vitivinicoles (O.N.V.P.V.) de Roumanie. Le R.P.V. est créé à la demande de l'Union Européenne et devient fonctionnel en 2007. À la base, il y a les déclarations des propriétaires de parcelles viticoles collectées entre l'année 2003 et l'année 2006. Chaque parcelle viticole se caractérisait d'un code d'identification unique, de la surface (en hectares), de la date de la plantation (mois et année), de(s) cépage(s) cultivé(s) et de l'emplacement de la parcelle par localité, commune et département.

Pour les départements où il y a eu de la reconversion de cépages, il a été distingué deux groupes de parcelles

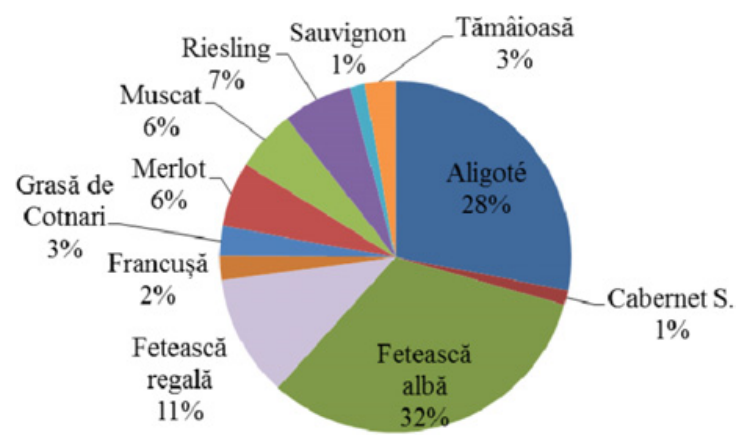

Figure 3. La structure de la surface des 10 premiers cépages cultivés dans la Région de Nord-Est en 2006 (Auteur : ENIA F. ; Données : ONVPV).

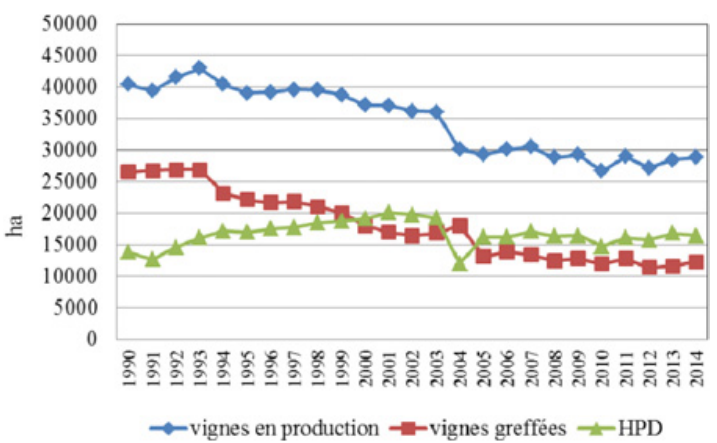

Figure 4. Evolution des surfaces en vignes greffées et en HPD dans la Région de Nord-Est pendant la période 1990-2014 (carte d'auteur ; Source de données : Institut National de Statistique).

pour chaque département : les parcelles existantes en terrain au niveau de l'année 2006 et les parcelles qui ont été reconverties pendant la période 2007-2015. Dans un premier temps la surface totale par cépages des deux groupes de parcelles a été classée en fonction de la couleur de la bais du raisin. Ensuite, pour chacune des classes il a été mis en évidence les cépages. Pour vérifier la couleur de la bais du raisin, la base de données de l'Organisation Internationale de la Vigne et du Vin (O.I.V.), sur la Roumanie, a été interrogée [6]. Dans un deuxième temps, la surface totale par cépages des deux groupes de parcelles a été classée en surface détenue par les cépages internationaux, respectivement en surface détenue par les cépages non-internationaux. Pour chacune des 2 dernières classes on mettra en évidence les cépages. Ayant comme modèle la définition donnée par Claude Gilois en mars 2016, le groupe des cépages dits internationaux correspond à « un groupe de sept cépages (Cabernet sauvignon, Merlot, Syrah, Pinot noir, Grenache, Chardonnay, Sauvignon blanc) choisis par les pays du Nouveau Monde pour développer leur viticulture et qui constituent $28 \%$ de l'encépagement mondial »[7]. En ce qui concerne la parcelle cultivée avec deux ou plus de deux cépages, le calcul de la surface pour chaque cépage a supposé l'application du pourcentage détenue de la surface totale de la parcelle. Dans le cas où, la somme des pourcentages détenus par les cépages de la parcelle dépassaient ou était sous $100 \%$, la parcelle était exclue de l'étude. Pour la parcelle viticole contenant des hybrides et de la vigne noble, en même temps, seulement la surface en vigne noble a été prise en compte. Les résultats sortis de la comparaison entre les 
2006

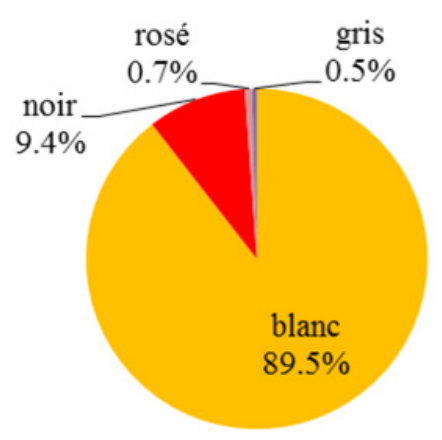

2007-2015

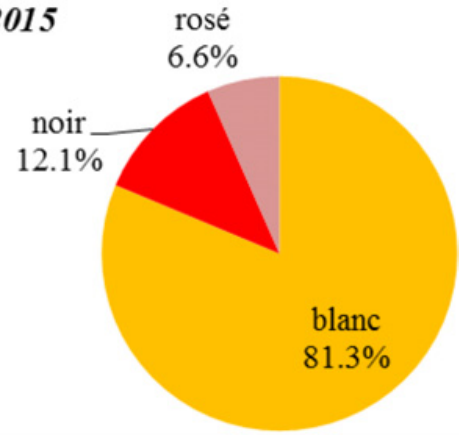

Figure 5. Iaşi- Structure de la surface viticole en fonction de la couleur de la baie en 2006 (en haut) et pour les parcelles nouveau plantées avec la mesure reconversion entre 2007-2015 (en bas) (Source de données brutes: ONVPV).

deux groupes ont été analysées à l'aide des entretiens avec des viticulteurs et avec des chercheurs, des données appartenant à l'Agence des Paies et d'Intervention pour l'Agriculture (A.P.I.A.) de Roumanie et des données sur les paramètres météorologiques de la Région de Nord-Est.

\section{Résultats}

La production de vins de la région se dirige principalement vers les vins blancs et de façon exceptionnelle vers les vins rouges [5] obtenus des cépages autochtones comme par exemple : Grasă de Cotnari, Fetească albă, Frâncuşă, Busuioacă de Bohotin, Zghihară de Huşi, Fetească neagră etc. Des cépages européens sont présents aussi : Aligoté, Riesling italien, Sauvignon, Cabernet Sauvignon, Merlot etc [3]. Les vins y obtenus sont en mode naturel des vins doux et couvrent une large variété, en commencent avec les vins de table et en finissant avec les vins à dénomination d'origine contrôlée. Les vins sont légers avec un contenu alcoolique modéré et avec une acidité qui peut arriver à 11-12 g/l d'acide tartrique. La production de raisins de table est faible [5] et utilisée pour satisfaire les besoins de la marchée locale. Parmi les cépages cultivés, il y a Chasselas doré et Perla de Csaba [3].

La figure 3 montre la structure des 10 premiers cépages comme surface dans la région, en 2006. Ces-ci couvrent plus de 8000 ha. En surfaces importantes sont cultivés la Fetească albă et l'Aligoté. Ces-ci sont suivis à distance par Fetească regală. Les cépages avec la baie noire sont représentés seulement par Cabernet Sauvignon et par Merlot qui donnent des vins supérieurs pour la région. Il est à remarquer la présence des cépages qui peuvent donnée des vins avec beaucoup d'arômes : Tămâioasă et Muscat Ottonel.
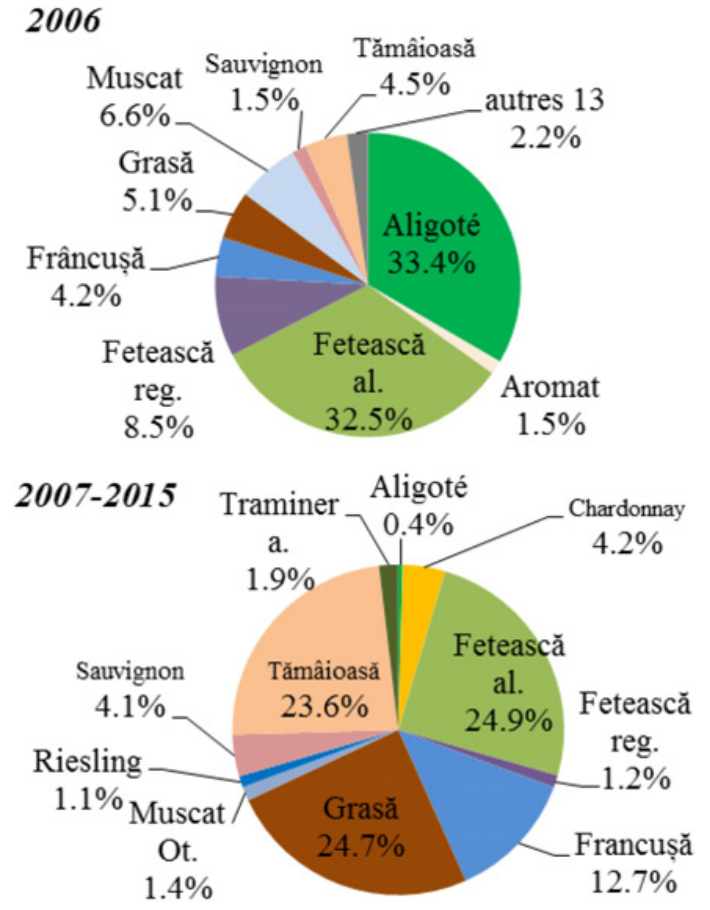

Figure 6. Iaşi- Structure de la surface viticole pour les cépages à baie blanc en 2006 (en haut) et pour les cépages à baie blanc plantées avec la mesure de reconversion entre 2007-2015 (en bas) (Source de données brutes: ONVPV).

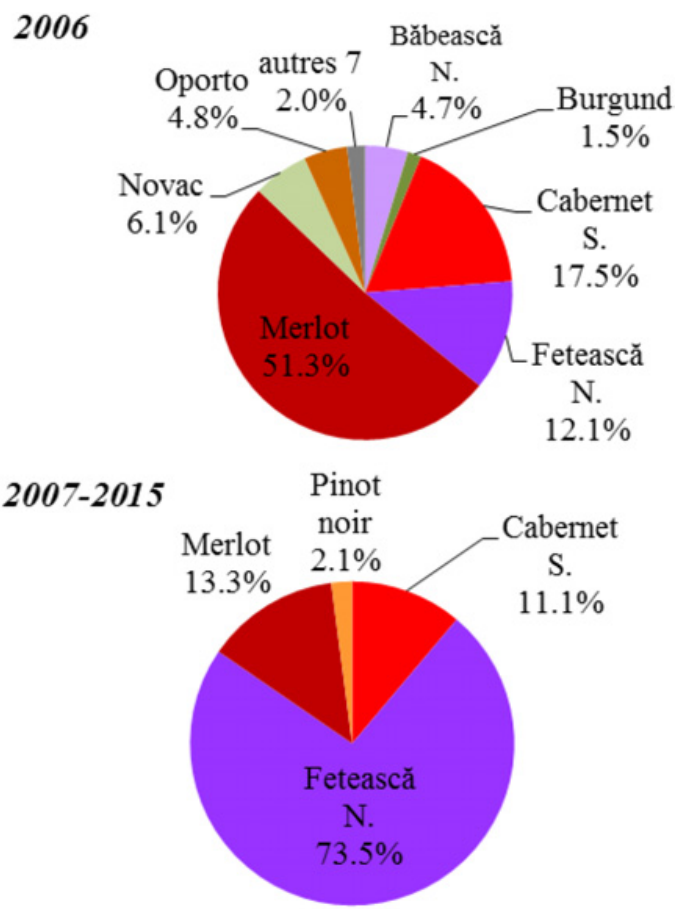

Figure 7. Iaşi- Structure de la surface viticole pour les cépages à baie noir en 2006 (en haut) et pour les cépages à baie noir plantées avec la mesure de reconversion entre 2007-2015 (en bas) (Source de données brutes : ONVPV).

Après la chute du régime communiste en 1989, le secteur vitivinicole de Roumanie, aussi comme autres secteurs d'activité, a connu beaucoup des changements. Par la Loi no. 18 de 1991 du Fond Foncier, on redonne aux anciens propriétaires les terrains prises pendant le processus de collectivisation de l'agriculture commencé 


\section{6}

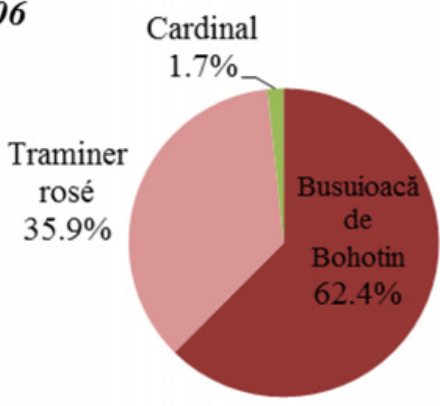

2007-2015

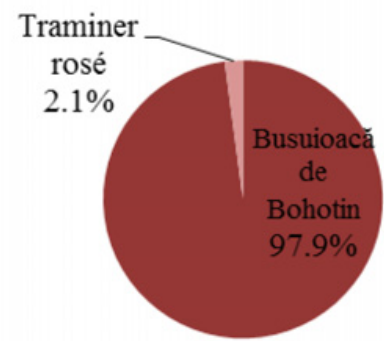

Figure 8. Iaşi- Structure de la surface viticole pour les cépages à baie rosé en 2006 (en haut) et pour les cépages à baie rosé plantées avec la mesure de reconversion entre 2007-2015 (en bas) (Source de données brutes : ONVPV).

2006

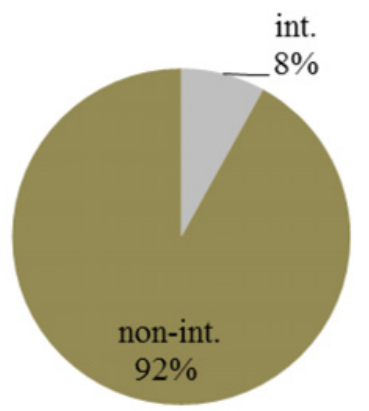

2007-2015

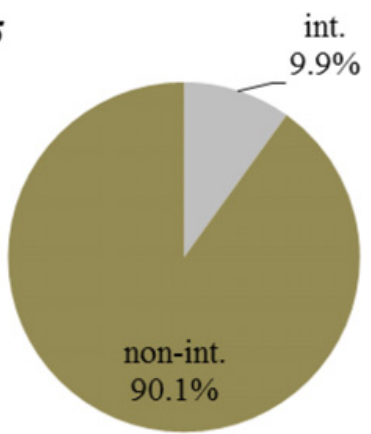

Figure 9. Iaşi- Structure de la surface viticole en cépages internationaux et non-internationaux en 2006 (en haut) et pour les cépages plantées avec la mesure de reconversion entre 20072015 (en bas) (Source de données brutes : ONVPV).

en 1949. Des importantes surfaces en vignes nobles des coopératives agricoles de production ou des entreprises agricoles d'état sont divisées et passent dans la propriété des petits propriétaires. La période d'après 1989 se caractérise par une augmentation importante des prix pour les traitements phytosanitaires et une baisse du prix du kilogramme de raisin. Les conditions économiques difficiles et la perte partielle des connaissances vitivinicoles
2006

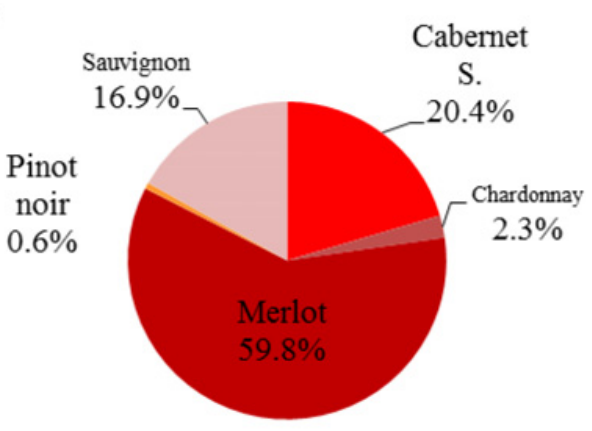

2007-2015

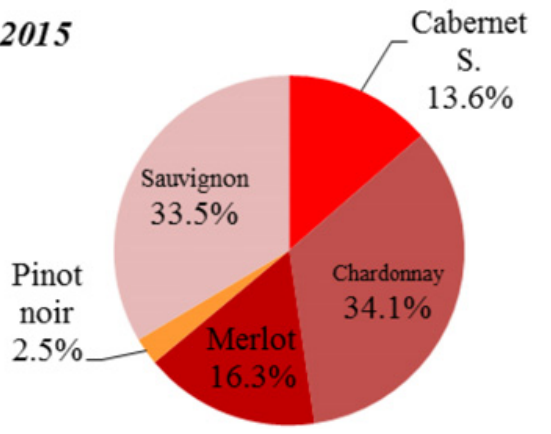

Figure 10. Iaşi- Structure de la surface viticole en cépages internationaux en 2006 (en haut) et pour les cépages plantées avec la mesure de reconversion entre 2007-2015 (en bas) (Source de données brutes: ONVPV).

2006

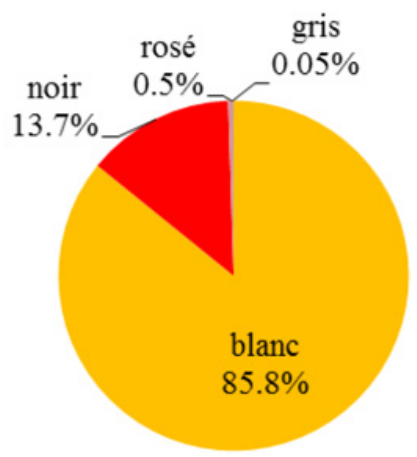

2007-2015

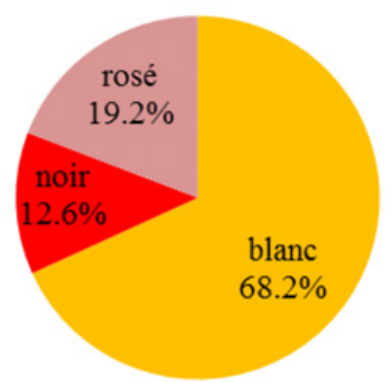

Figure 11. Vaslui- Structure de la surface viticole en fonction de la couleur de la baie en 2006 (en haut) et pour les parcelles nouveau plantées avec la mesure reconversion entre 2007-2015 (en bas) (Source de données brutes : ONVPV).

conduisent à la dégradation des vignes nobles qui des fois sont remplacées avec des hybrides producteurs directs. La détérioration du patrimoine en vignes nobles peut-être observée dans la Fig. 4.

La mesure de restructuration et reconversion des vignobles est stipullée à l'Article 103 octodecies du Règlement (CE) no. 1234/2007 « portant organisation 


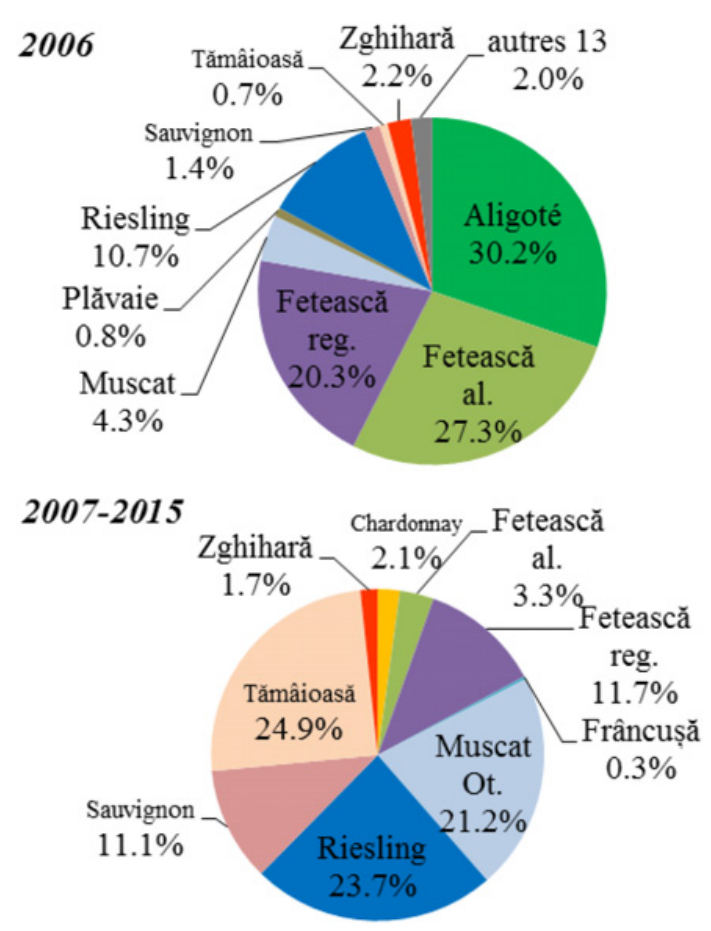

Figure 12. Vaslui- Structure de la surface viticole pour les cépages à baie blanc en 2006 (en haut) et pour les cépages à baie blanc plantées avec la mesure de reconversion entre 2007-2015 (en bas) (Source de données brutes : ONVPV).

commune des marchés dans le secteur agricole et dispositions spécifiques en ce qui concerne certains produits de ce secteur» [8] et dans le Réglement (UE) no. 1308/2013 « portant organisation commune des marchés des produits agricoles » [9]. Ayant comme objectif d'accroître la competitivité entre les viticulteurs, la mesure peut-être accédée seulement par les états qui sumissent un « inventaire de leur potentiel de production ». Cet inventaire qui doit être envoyé annuellement, concerne les pays ayant plus de 500 ha de vignes des variétés à raisins de cuve ([8], Article 185 bis, [9] Article 145), considérées appartant à l'espèce Vitis vinifera ou provient d'un croisement entre ladite espèce et d'autres espèces du genre Vitis ([8], Article 120 bis).

En Roumanie, la mesure est transcrite à partir de 2009 dans le Plan National de Support de la Roumanie dans le Secteur Vitivinicole (PNSRSV). Ceci contient aussi des autres mesures pour lesquelles la Roumanie a appliqué à partir de 2007 : l'assurance de la récolte, l'investissement dans des entreprises, la distillation des sous-produits et la promotion des vins, le système de payement unique (non-disponible à partir de 2014), la récolte avant le murissement (non-disponible à partir de 2014), les fonds mutuelles (non-disponible à partir de 2014) [10]. Conformément au PNSRSV, « la reconversion des cépages consiste en changer les cépages existantes dans la culture avec des cépages supérieurs de point de vue qualitatif ou avec des clones des mêmes cépages $\gg$. La plantation peut être faite seulement avec des cépages nobles à raisins de cuve. La plantation peut-être choisie sur le même emplacement ou sur un emplacement équivalent en surface. Le support financière ne peut pas dépasser $75 \%$ des coûts et la base de calcul est fournie par le devis de dépenses pour chaque catégorie de travaux [11]. La mesure de reconversion et de restructuration des vignoble comprend 3 autres activités : le remplacement

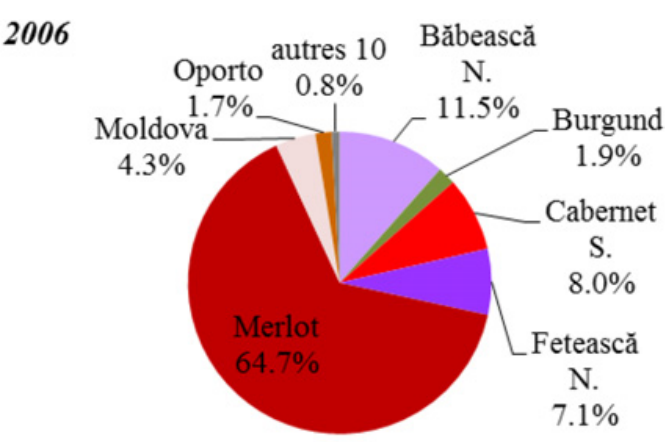

$2007-2015$

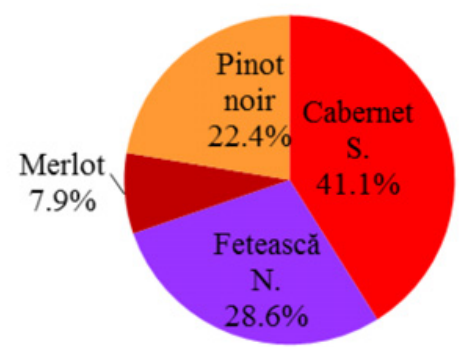

Figure 13. Vaslui- Structure de la surface viticole pour les cépages à baie noir en 2006 (en haut) et pour les cépages à baie noir plantées avec la mesure de reconversion entre 2007-2015 (en bas) (Source de données brutes : ONVPV).

2006

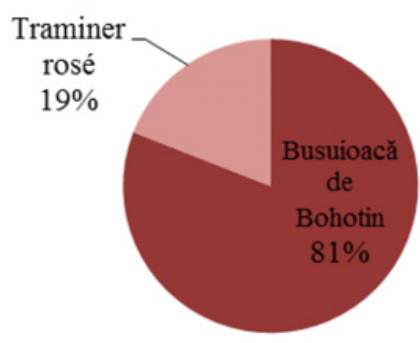

\section{7-2015}

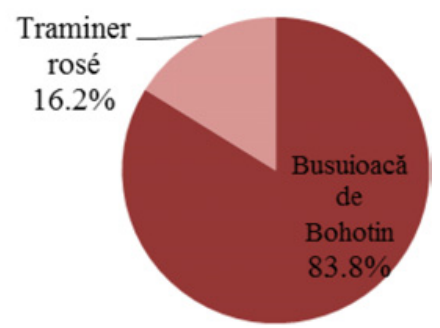

Figure 14. Vaslui- Structure de la surface viticole pour les cépages à baie rosé en 2006 (en haut) et pour les cépages à baie rosé plantées avec la mesure de reconversion entre 2007-2015 (en bas) (Source de données brutes : ONVPV).

des parcelles, la modernisation des exploitations viticoles, la replantation des parcelles viticoles si l'arrachage a à la base des raisons sanitaires ou phytosanitaires [10].

Des 6 départements de la Région de Nord-Est il y a eu de la reconversion de cépages dans 3 départements : Bacău, Iaşi et Vaslui.

\subsection{Les vignobles de laşi}

Dans le département de Iaşi, les vignes se trouvent plutôt dans les deux vignobles, Cotnari et Iaşi. Il y a aussi deux 


\section{6}

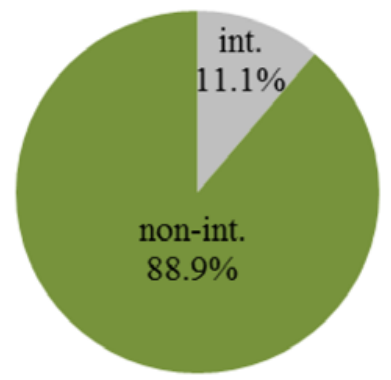

$2007-2015$

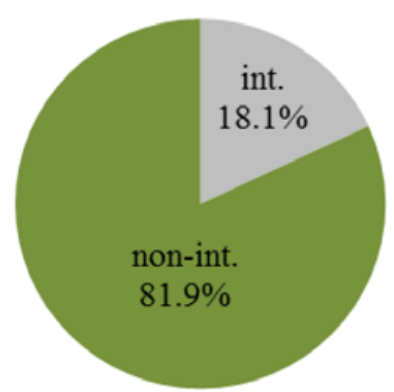

Figure 15. Vaslui- Structure de la surface viticole en cépages internationaux et non-internationaux en 2006 (en haut) et pour les cépages plantées avec la mesure de reconversion entre 2007-2015 (en bas) (Source de données brutes : ONVPV).

centres viticoles indépendants, Plugari et Probota et des nombreuses plantations petites dans les cours des citoyens à la campagne, à l'extérieure de ces vignobles et centres viticoles. Les dénominations d'origine contrôlées sont en nombre de trois : Cotnari, Iaşi et Bohotin. La surface totale des cépages nobles, sauf cépages à raisin de table, dépassait 4700 ha en 2006. Pendant la période 2007-2015, la mesure de reconversion a été appliquée pour un peu plus de 1672,3 ha.

\subsubsection{Profil des vignobles}

See Figs. 5-8

\subsubsection{La présence des cépages internationaux}

See Figs. 9 and 10.

\subsection{Les vignobles de Vaslui}

Les vignes de ce département font partie du vignoble de Huşi, de la petite vignoble Les Collines de Tutova (deux centres viticoles sur trois) et du centre viticole indépendant Vaslui. À l'extérieur de ces-ci se trouvent des petites surfaces en vignes (plutôt des hybrides) dans les cours des citoyens de la campagne. Huşi, c'est la seule dénomination d'origine contrôlée. Sauf la surface des cépages donnant des raisins de table, la surface viticole en cépages nobles dépasse 2700 ha, en 2006. La conversion de cépages a été appliquée pour 959,3 ha.
2006

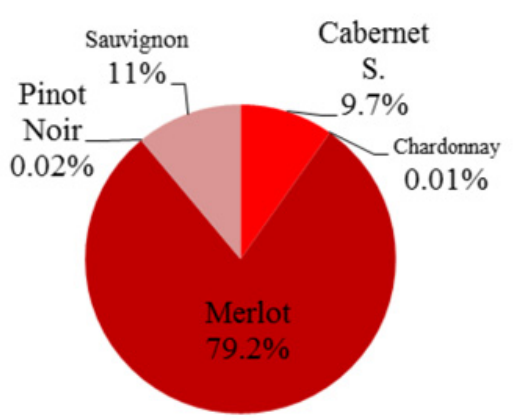

\section{$2007-2015$}

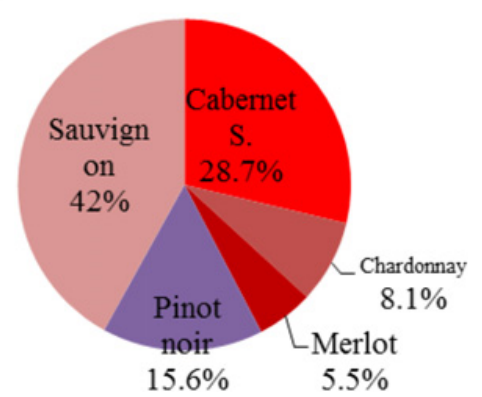

Figure 16. Vaslui- Structure de la surface viticole en cépages internationaux en 2006 (en haut) et pour les cépages plantées avec la mesure de reconversion entre 2007-2015 (en bas) (Source de données brutes : ONVPV).

2006

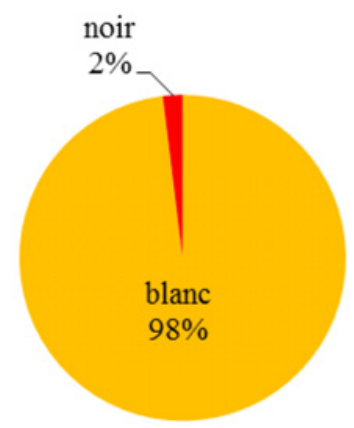

$2007-2015$

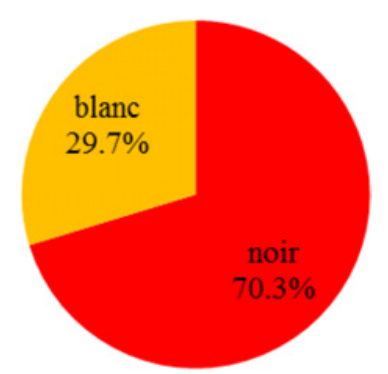

Figure 17. Bacău- Structure de la surface viticole en fonction de la couleur de la baie en 2006 (en haut) et pour les parcelles nouveau plantées avec la mesure reconversion entre 2007-2015 (en bas) (Source de données brutes : ONVPV).

\subsubsection{Profil des vignobles}

See Figs. 11-14.

\subsubsection{La présence des cépages internationaux}

See Figs.15 and 16. 


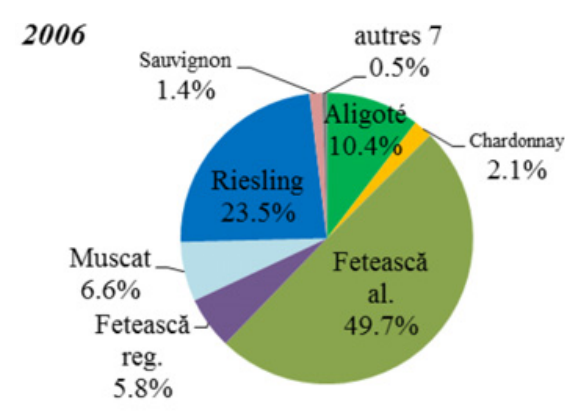

$2007-2015$

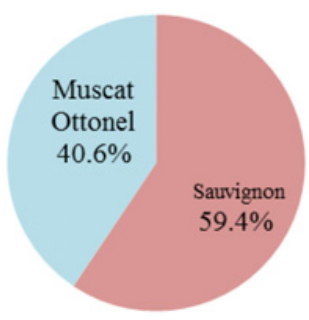

Figure 18. Bacău- Structure de la surface viticole pour les cépages à baie blanc en 2006 (en haut) et pour les cépages à baie blanc plantées avec la mesure de reconversion entre 2007-2015 (en bas) (Source de données brutes : ONVPV).

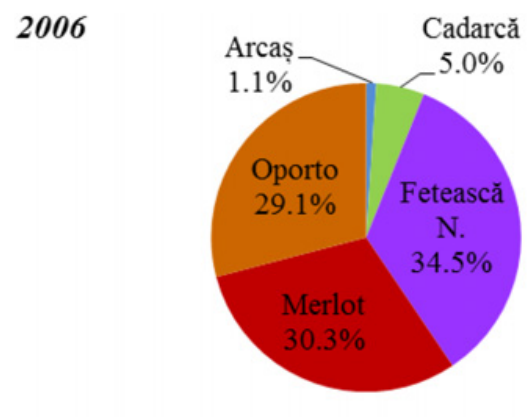

$2007-2015$

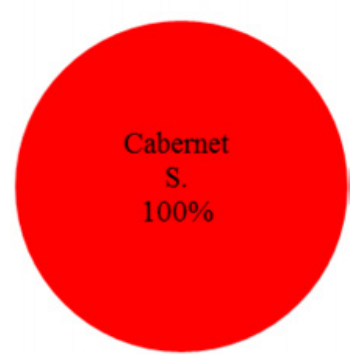

Figure 19. Bacău- Structure de la surface viticole pour les cépages à baie noir en 2006 (en haut) et pour les cépages à baie noir plantées avec la mesure de reconversion entre 2007-2015 (en bas) (Source de données brutes : ONVPV).

\subsection{Les vignobles de Bacău}

La surface en vignes noble dans le département de Bacău était d'à peu près 1000 ha en 2006. La surface qui a été soumise à la conversion de cépages a été la plus petite des trois départements, en arrivant à 28,4 ha seulement.

\subsubsection{Profil des vignobles}

See Figs. 17-19.

\subsubsection{La présence des cépages internationaux}

See Figs. 20 and 21.
2006

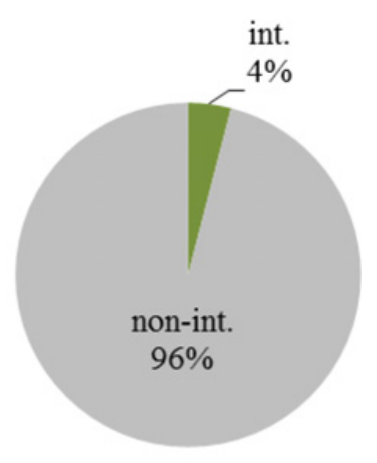

$2007-2015$

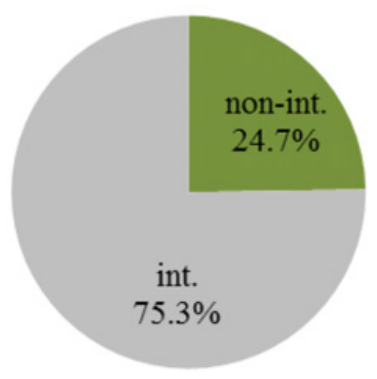

Figure 20. Bacău- Structure de la surface viticole en cépages internationaux et non-internationaux en 2006 (en haut) et pour les cépages plantées avec la mesure de reconversion entre 2007-2015 (en bas) (Source de données brutes : ONVPV).

\section{6}

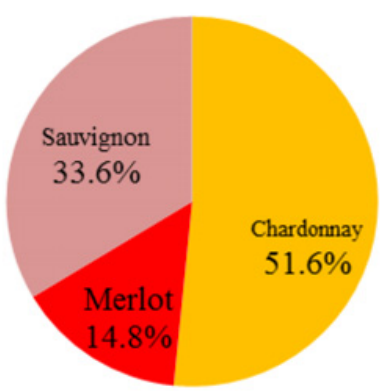

$2007-2015$

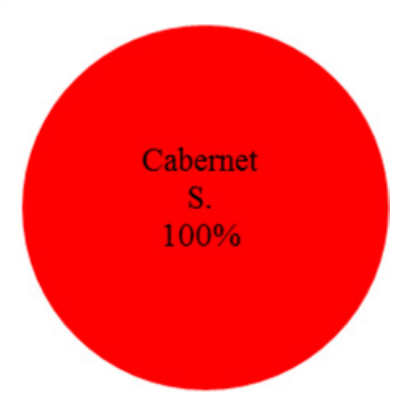

Figure 21. Bacău- Structure de la surface viticole en cépages internationaux en 2006 (en haut) et pour les cépages plantées avec la mesure de reconversion entre 2007-2015 (en bas) (Source de données brutes : ONVPV).

\section{Conclusion}

La mesure de conversion des cépages amène des nombreux bénéfices à la viticulture de la Région de Nord-Est de la Roumanie en aidant à mieux répondre aux demandes du consommateur du vin. Même si la conversion en cépages à 
baie blanche a été plus importante, les cépages à baie rouge et rosée occupent de plus en plus de surface et annoncent un possible début de l'échange de profil soutenu par le climat qui est plus doux. Quand même, la conservation des cépages traditionnelles est observée dans le cas du département de Iaşi où dans le Vignoble de Cotnari la conversion a été faite plutôt avec les quatre cépages : Grasă de Cotnari, Frâncuşă, Fetească albă et Tămâioasă. Egalement, les propriétaires de vignes ont choisi des cépages reconnus de donner des vins doux naturels, sauf que, à partir de ces cépages, ils font plus souvent des vins secs ou demi-secs avec beaucoup d'arômes. Dans tous les départements, un ou plusieurs des cépages suivants a connu des augmentations de surface : Tămâioasă românească, Muscat Ottonel, Busuioacă de Bohotin etc. En continuation, les cépages non-internationaux et dans la plupart des cas, les cépages locaux sont préférés dans les départements à longue tradition viticoles, Iaşi et Vaslui.

\section{References}

[1] Ministère de l'Agriculture et du Développement Rurale (MADR) - La Région de Développement de Nord-Est http://www.mdrl.ro/_documente/ regiuni/1.NE_ro.pdf

[2] OIRPOSDRU NORD-EST - Plan de Développement Régional 2007-2013 Nord-Est http://www.fsen ordest.ro/Download/doc_prog/Planuldedezvo ltareregionala-analizaecNE.pdf

[3] V. D. Cotea, Vignobles et vins de Roumanie, (2003)

[4] ONVPV, Cahier des charges pour la production et la commercialisation du vin à dénomination d'origine contrôlée “Cotnari ”- http://www.onvpv.ro/ sites/default/files/pdfs/caiet_de_sarcini _doc_cotnari_238ro_0.pdf

[5] ONVPV, Cahier des charges pour la production et la commercialisation du vin à indication géographique «Les collines de la Moldavie 》- http://www. onvpv.ro/sites/default/files/pdfs/caiet_ de_sarcini_ig_dealurile_moldovei_282ro. pdf

[6] OIV, Base de données et statistique http://www. oiv.int/fr/bases-de-donnees-et-statistiq ues $/$ bases-de-donneesşbdd=Cepage

[7] C. Gilois, Peut-on faire des grands vins dans les cépages internationaux ?, 2016 http://voyage svinsdumonde.20minutes-blogs.fr/archive/ 2016/02/26/cepages-internationaux-partie -1 -de-2.html

[8] RÈGLEMENT (CE) No 1234/2007 DU CONSEIL http://eurlex.europa.eu/LexUriServ/LexUri Serv. do?uri=CONSLEG : 2007R1234 : $20100501:$ FR : PDF

[9] RÈGLEMENT (UE) No 1308/2013 DU PARLEMENT EUROPÉEN ET DU CONSEIL lex. europa.eu/LexUriServ/LexUriServ.do?uri= OJ :L:2013: $347: 0671: 0854:$ fr :PDF

[10] Ministère de l'Agriculture et du Développement Rural - Programme National de Support de la Roumanie dans le secteur vitivinicole 2014 - 2018 http://www.madr.ro/horticultura/viticult ura-vinificatie.html

[11] Ministère de l'Agriculture et du Développement Rural, Le dépôt du Programme National de Support de la Roumanie dans le secteur vitivinicole 2014 2018 